\title{
saicar

\section{Comparison of bulk and pitcher-catcher targets for laser-driven neutron production}

L. Willingale, G. M. Petrov, A. Maksimchuk, J. Davis, R. R. Freeman et al.

Citation: Phys. Plasmas 18, 083106 (2011); doi: 10.1063/1.3624769

View online: http://dx.doi.org/10.1063/1.3624769

View Table of Contents: http://pop.aip.org/resource/1/PHPAEN/v18/i8

Published by the AIP Publishing LLC.

\section{Additional information on Phys. Plasmas}

Journal Homepage: http://pop.aip.org/

Journal Information: http://pop.aip.org/about/about_the_journal

Top downloads: http://pop.aip.org/features/most_downloaded

Information for Authors: http://pop.aip.org/authors

\section{ADVERTISEMENT}

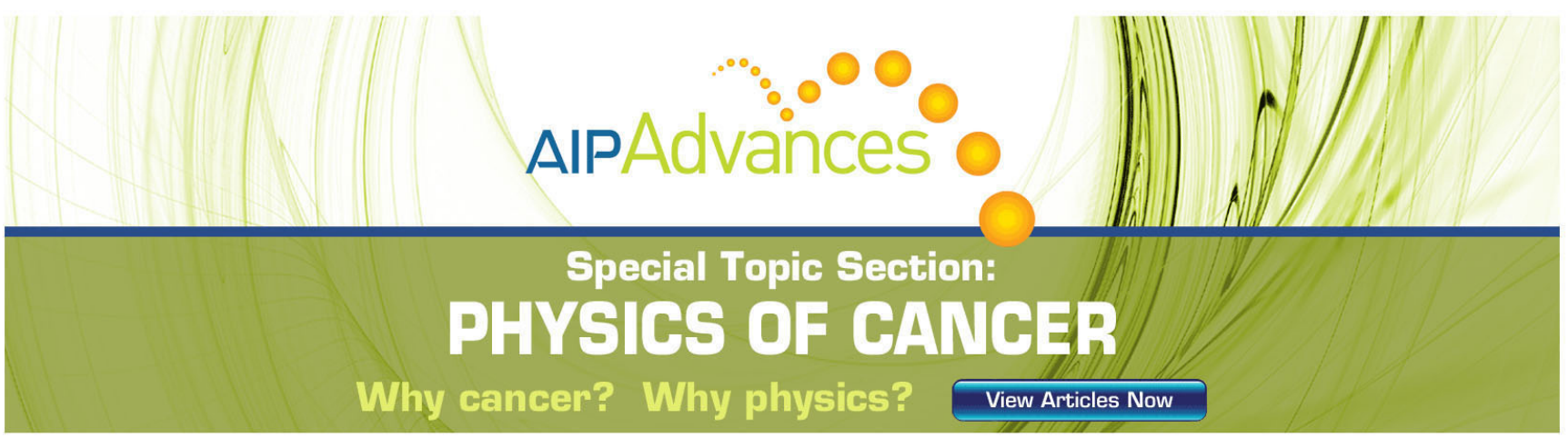




\title{
Comparison of bulk and pitcher-catcher targets for laser-driven neutron production
}

\author{
L. Willingale, ${ }^{1}$ G. M. Petrov, ${ }^{2}$ A. Maksimchuk, ${ }^{1}$ J. Davis, ${ }^{2}$ R. R. Freeman, ${ }^{3}$ A. S. Joglekar, ${ }^{1}$ \\ T. Matsuoka, ${ }^{1}$ C. D. Murphy, ${ }^{3}$ V. M. Ovchinnikov, ${ }^{3}$ A. G. R. Thomas, ${ }^{1}$ L. Van Woerkom, ${ }^{3}$ \\ and K. Krushelnick ${ }^{1}$ \\ ${ }^{1}$ Center for Ultrafast Optical Science, University of Michigan, 2200 Bonisteel Boulevard, Ann Arbor, \\ Michigan 48109, USA \\ ${ }^{2}$ Naval Research Laboratory, Plasma Physics Division, 4555 Overlook Avenue SW, Washington, DC 20375, \\ USA \\ ${ }^{3}$ Department of Physics, The Ohio State University, Columbus, Ohio 43210, USA
}

(Received 12 May 2011; accepted 20 July 2011; published online 19 August 2011)

Laser-driven $\mathrm{d}(d, n)-{ }^{3} \mathrm{He}$ beam-target fusion neutron production from bulk deuterated plastic (CD) targets is compared with a pitcher-catcher target scheme using an identical laser and detector arrangement. For laser intensities in the range of $(1-3) \times 10^{19} \mathrm{~W} \mathrm{~cm}^{-2}$, it was found that the bulk targets produced a high yield $\left(5 \times 10^{4}\right.$ neutrons per steradian) beamed preferentially in the laser propagation direction. Numerical modeling shows the importance of considering the temperature adjusted stopping powers to correctly model the neutron production. The bulk CD targets have a high background target temperature leading to a reduced stopping power for the deuterons, which increases the probability of generating neutrons by fusion. Neutron production from the pitcher-catcher targets was not as efficient since it does not benefit from the reduced stopping power in the cold catcher target. Also, the inhibition of the deuteron acceleration by a proton rich contamination layer significantly reduces the pitcher-catcher neutron production. (c) 2011 American Institute of Physics.

[doi:10.1063/1.3624769]

\section{INTRODUCTION}

Energetic ion beams accelerated in relativistically intense laser interactions have a wide spectrum of possible applications in the scientific, medical, and engineering communities. One such use of these high energy ion beams could be a compact, high-brightness, short duration, and relatively low-cost neutron source. These attributes are advantageous qualities for applications such as fast neutron radiography, active nuclear interrogation, and fusion research. Determining the ion dynamics in laser-plasma interactions can be difficult because global electromagnetic fields may influence the ions as they leave the target, meaning that an externally measured spectra may not be representative of conditions within the target. The $\mathrm{d}(d, n)-{ }^{3} \mathrm{He}$ fusion reaction produces $2.45 \mathrm{MeV}$ center-of-mass energy neutrons and through the precise measurement of the neutron energy, information about the energetic ion beam within the target can be deduced. ${ }^{1-9}$ Neutron measurements have inferred the deuteron acceleration direction at the front surface of deuterated targets for a high contrast ratio ${ }^{3}$ or a low contrast ratio where ion shock acceleration was diagnosed. ${ }^{9}$

The deuteron beam characteristics influence the generated neutron angular distribution and spectra. For beam-target fusion, the $2.45 \mathrm{MeV}$ neutrons are upshifted to higher energies in a beam due to the directional deuteron beam momentum. ${ }^{6,10}$ Therefore, a laser generated neutron source has a good potential for neutron radiography. If the laser interacts directly with the deuterated material, the ponderomotive force of the laser drives the electrons into the target, setting up a charge imbalance, which accelerates ions into the tar- get $^{11,12}$ and at highest intensities via an electrostatic shock. ${ }^{13}$ If the intensity is great enough, the ponderomotive force drives a shock into the target. ${ }^{9}$ Fusion reactions can occur as the accelerated deuterons move through the deuterated targets, whether it be a solid target, ${ }^{1-3}$ clusters,,${ }^{4,5}$ gas, ${ }^{7}$ or a droplet. ${ }^{8}$

Alternatively a secondary deuterated target, known as a "catcher", can intercept the pre-accelerated deuteron beam. ${ }^{7,8,14}$ This is known as the "pitcher-catcher" (P-C) method and has the advantage of naturally selecting a collimated ion beam. Here, the deuteron beam is accelerated from thin foil targets coated with a deuterated plastic via either a front or rear side acceleration (RSA) mechanism. Deuterons can be accelerated from the rear surface of the thin foil target via the target normal sheath acceleration (TNSA) mechanism ${ }^{15}$ or from the front surface through a ponderomotive or shock acceleration mechanism. ${ }^{11-13}$ In the TNSA regime, hot electrons generated by the laser move through the target and out into the vacuum at the rear of the target. The charge separation leads to a large electrostatic sheath field, which ionizes the rear surface and accelerates a well collimated ion beam. If the front side accelerated ions have enough energy, they can pass through the target, emerge from the rear side, and also move into the vacuum joining those generated via TNSA. The relative effectiveness of each of these mechanisms for deuterons has been found to be approximately equal under the experimental conditions to be considered here. ${ }^{16}$

Other neutron generation schemes use ${ }^{3} \mathrm{~T}(d, n)$ fusion ${ }^{17}$ or more exotic reactions such as ${ }^{7} \mathrm{Li}(p, n)$ (Refs. 18-20), ${ }^{7} \mathrm{Li}(d, x n),{ }^{21}$ other light ion reactions, ${ }^{22}$ or even $(\mathrm{p}, \mathrm{n})$ reactions with high $\mathrm{Z}$ materials such as lead. ${ }^{23}$ The reaction cross-sections and center-of-mass energy for the neutrons 
produced may be even more favorable than the $\mathrm{d}(d, n)-{ }^{3} \mathrm{He}$ considered here, but the same principles will also apply to these reactions. The presence of carbon in a deuterated target can also make a contribution to the neutron yield. ${ }^{24}$

We present a direct comparison between $d$ - $d$ neutron production for two types of target; a bulk deuterated plastic target and a thin foil interaction which accelerates a deuteron beam, the pitcher, into a deuterated plastic catcher. The experimental setup is presented in Sec. II and the numerical methods in Sec. III. Then, the results of the investigation are given in Sec. IV. The effect of the background target temperature is found to be important for calculating the neutron production due to the strong dependance of deuteron stopping power with background target temperature. Section V summarizes the work along with suggestions for improvements to the next generation of laser-driven neutron sources.

\section{EXPERIMENT}

The experiments were performed using the T-cubed laser at the University of Michigan, which is a chirped pulse amplification (CPA) hybrid Ti:sapphire/Nd:phosphate glass system (central wavelength of $1.053 \mu \mathrm{m}$, linearly polarized). The on target energy was up to $\mathcal{E}_{L}=6 \mathrm{~J}$ in a full-width halfmaximum (FWHM) pulse length of 400 fs. An $f / 2.7$ off-axis parabolic mirror focused the pulse to a $5 \mu \mathrm{m}$ diameter FWHM spot, giving a peak vacuum intensity of $I_{0}$ $=2.6 \times 10^{19} \mathrm{~W} \mathrm{~cm}^{-2}$ (corresponding to a normalized vector potential of $a_{0}=4.5$ ). The angle of incidence onto the target was $22.5^{\circ}$ with respect to the incoming p-polarized laser beam. The nanosecond energy contrast due to amplified spontaneous emission (ASE) was of the order $10^{-5}$.

The bulk targets were made from compressed, deuterated polyethylene with thickness of $1.5 \pm 0.5 \mathrm{~mm}$. The P-C targets were $13 \mu \mathrm{m}$ Mylar foils with a deuterated layer $(\sim 1 \mu \mathrm{m})$ deposited on either the front, rear, or both target surfaces using a solution of deuterated polystyrene, CD. It is expected that the usual hydrocarbon contaminant layer present on the typical experimental foils would have formed over the top of the CD layer. Deuterons are accelerated from either surface, ${ }^{16,25}$ from the rear surface of the thin foil target via the TNSA mechanism ${ }^{15}$ or from the front surface through a ponderomotive acceleration mechanism. ${ }^{11,12}$ The catcher was a sheet of deuterated polystyrene, approximately $0.5 \mathrm{~mm}$ thick, in the target normal direction and would intercept a deuteron beam with an angular divergence of $45^{\circ}$. A $1.5 \mathrm{~mm}$ diameter hole through the center of the catcher allowed a line of sight to the Thomson parabola spectrometer, which measures the ion spectra in the target normal direction.

To detect the neutrons, three scintillators (EJ-204 plastic) coupled using light guides to photo-multiplier tubes (PMTs) were placed on the horizontal plane of the interaction. The laser propagation direction, $\theta=0^{\circ}$, defines the angular positions of the scintillator detectors as shown in Figure 1. Neutron spectra are determined using a time-offlight (ToF) method. The detector response function was calibrated using a d-d neutron generator. The Monte-Carlo code MCNP 5 (Ref. 26) was run to calculate the attenuation in the shielding surrounding the detector.

\section{NUMERICAL MODELING}

A series of simulations are performed to match the conditions of the experiments using a two-dimensional, fully relativistic, electromagnetic particle-in-cell (PIC) model. ${ }^{10,27}$ The laser beam parameters used are similar to the experiment, albeit at normal incidence, with varying peak laser intensity $I_{0}=(1-3) \times 10^{19} \mathrm{~W} \mathrm{~cm}^{-2}$. The pre-plasma is modeled as having a profile which falls off exponentially with a characteristic scale length of $0.2 \mu \mathrm{m}$ (at $1 / e$ level) and a total of $3 \mu \mathrm{m}$ of pre-plasma. The flat top part of the target is a total of $7 \mu \mathrm{m}$ long and has an electron density of $200 n_{c}$, where $n_{c}$ is the critical electron density. For the front side CD targets, replicating the front side acceleration (FSA) of deuterons, the pre-plasma region and a further $1 \mu \mathrm{m}$ of the maximum plasma density consists of a CD plasma followed by $6 \mu \mathrm{m}$ of aluminum plasma. For the rear side CD targets, replicating the P-C RSA of deuterons, the pre-plasma region and a further $6 \mu \mathrm{m}$ is aluminum plasma followed by $1 \mu \mathrm{m}$ of $\mathrm{CD}$ and a $2 \mathrm{~nm}$ thick $\mathrm{H}_{2} \mathrm{O}$ contamination layer (this has been shown to influence the deuteron acceleration ${ }^{16,28}$ ).

The energy and angular distribution of neutrons are calculated with a 3D Monte-Carlo simulation code, which follows the transport of deuterons through a secondary target (catcher - note that the catcher could be the target itself as for the bulk target). ${ }^{29}$ During the deuteron transport, the

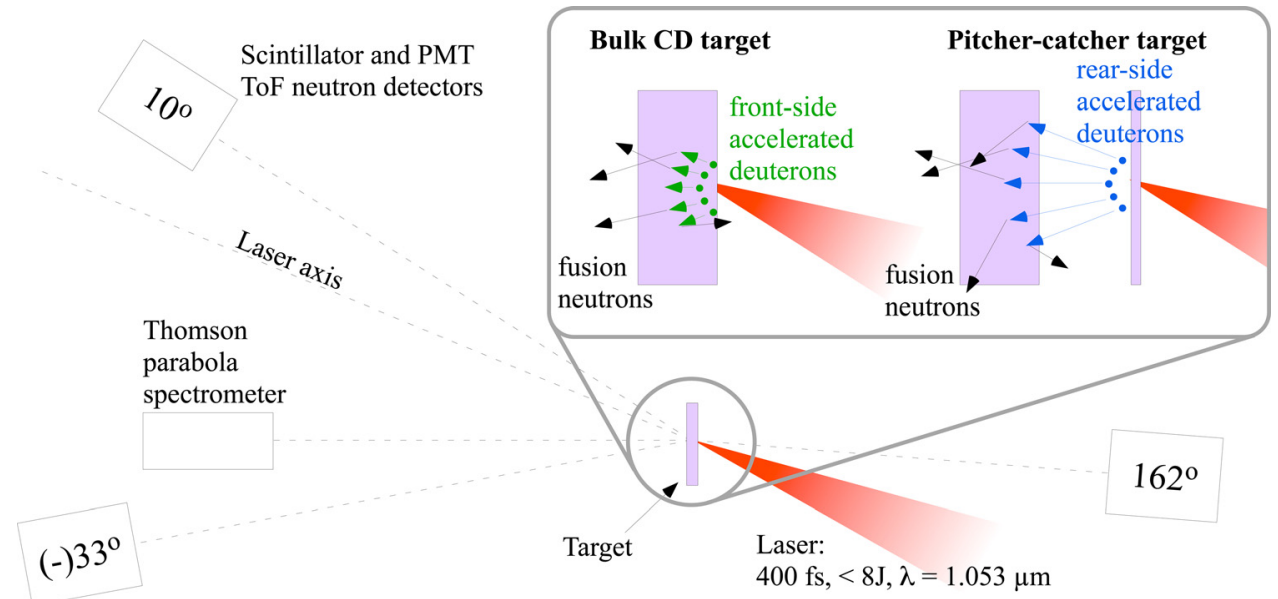

FIG. 1. (Color online) Schematic of the experiment showing the positions of the neutron detectors with respect to $\theta=0^{\circ}$, the laser propagation direction. The two targets types: (left) bulk CD targets from which the deuterons are accelerated into the target from the front surface to react with deuterons within the target itself, (right) pitcher-catcher targets, where deuterons are accelerated from thin foil targets (pitcher) and then collide with a thick deuterated target, the catcher, where the fusion reactions take place. 
neutron flux is calculated in specific directions of observation. The catcher is assumed to be sufficiently thick (a few $\mathrm{mm}$ ) so that even the most energetic deuterons are completely stopped inside. The ion and neutron distributions are assumed to be axi-symmetric with respect to the laser propagation direction. The ion transport is based on the continuous slowing down approximation ${ }^{30}$ using the concept of stopping power and angular scattering via small angle scattering in collisions with the atoms of the secondary target. Ions are launched one by one from the surface of the secondary target with energy and angle (with respect to the normal surface), known from the particle-in-cell model. The trajectory of the moving deuteron is presented as a series of straight lines and isolated binary collisions. Elastic collisions with the atomic nuclei determine the scattering. The energy loss is determined by bound electrons in the P-C (RSA) case, where the catcher is an isolated solid target, whereas in the bulk target (FSA), the stopping powers are modified according to the temperature of the target. The energy loss of ions in cold plasma is due to the bound electrons only, but with increasing target temperature, the material begins to ionize and the energy loss becomes dominated by free (unbound electrons). For a cold target, we follow the theory of Ziegler and Biersack $^{31,32}$ for bound electrons. They consider two limiting cases. At low projectile velocity, $v_{\text {ion }}<\alpha c Z_{i o n}^{\frac{2}{3}}$, the so-called Lindhard-Scharff-Schiott (LSS) theory is valid, ${ }^{32}$ and in the opposite case of high projectile velocity, the stopping power reduces to the Bethe formula. ${ }^{33}$ Here, $\alpha=1 / 137$ is the fine structure constant, $c$ is the speed of light, and $Z_{i o n}$ is the projectile charge. Interpolations formula connects the two regions. ${ }^{32}$ For free electrons, a similar situation exists: low and high projectile velocities, but the boundary between them is now defined by the velocity of thermal electrons, $v_{e, t h}=\sqrt{2 T_{e} / m_{e}}$, where $T_{e}$ is the electron temperature of the plasma. A comprehensive model for the stopping power by free electrons in the limiting cases $v_{\text {ions }} \ll v_{e, t h}, v_{\text {ions }} \gg v_{e, t h}$ and a smooth transition between them with useful analytical

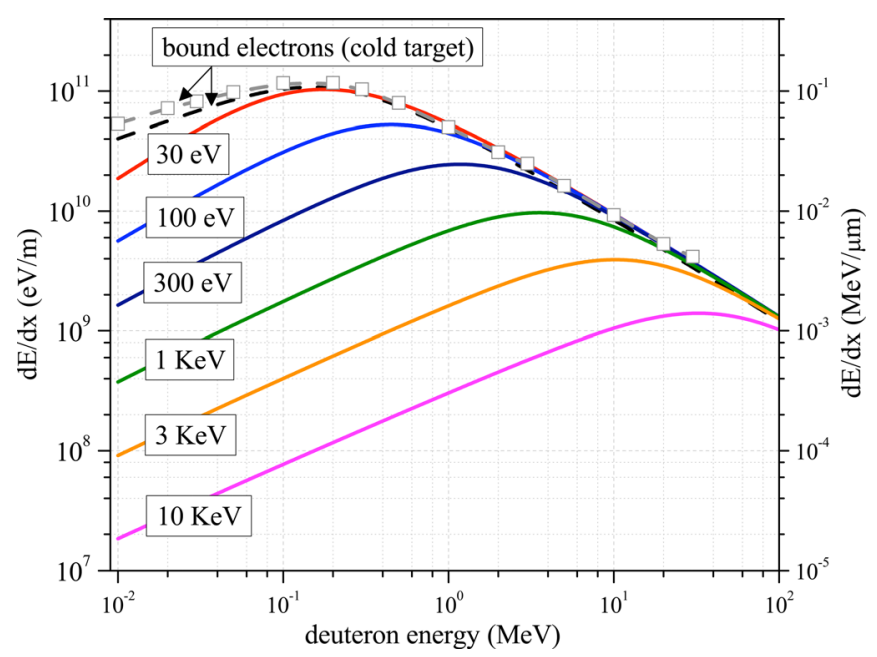

FIG. 2. (Color online) Stopping powers for deuterons in a cold CD target (gray dashed line and squares: Ref. 29, black dashed line: Ref. 30) and target with different background electron temperatures (solid lines) calculated according to Ref. 30. The density of CD is $\rho_{C D}=1.1 \mathrm{~g} \mathrm{~cm}^{-3}$ and the densities of carbon and deuterium atoms are $n_{C}=n_{D}=4.6 \times 10^{22} \mathrm{~cm}^{-3}$. expressions for arbitrary projectile velocities has been developed in Ref. 30. Figure 2 shows how the stopping power of a deuteron in $\mathrm{CD}$ varies with background temperature which are calculated using the methods described by Mehlhorn. ${ }^{30}$ For high deuteron energy, such that $v_{d} \gg v_{e, t h}$, the stopping power is independent of the background electron temperature, while in the opposite case of low deuteron energy, the stopping power depends strongly on the electron temperature. The effect of the target energy on non-thermal beam target fusion reactions has been studied and has demonstrated the theoretical enhancement of the gain for high target temperatures. ${ }^{17,34-36}$ As the background temperature of the target increases, the stopping power is reduced and therefore the deuterons will be able to travel further through the $\mathrm{CD}$, increasing the probability of a $\mathrm{d}(d, n)-{ }^{3} \mathrm{He}$ fusion reaction. The electron temperature profile into the target is assumed to be $T_{e}(z)=T_{0} \exp (-z[\mu \mathrm{m}] / 10)$ (Ref. 37) with $T_{0}$ being taken as the average electron temperature taken from the PIC simulations. For the simulated intensities $I=(1,2$, $3) \times 10^{19} \mathrm{~W} \mathrm{~cm}^{-2}$ and $T_{0}=(0.3,0.7,1.0) \mathrm{keV}$, it was found that the neutron yield was very sensitive to the parameter $T_{0}$ but not sensitive to the temperature profile inside the target.

The entire procedure per straight-line advance involves the following four steps: (i) subtract energy from the deuteron. The magnitude of the energy loss $\Delta E_{d}=S\left(E_{d}, T_{e}\right) \Delta L$ is determined by the stopping power $S\left(E_{d}, T_{e}\right)$ for the current deuteron energy $E_{d}$, local temperature $T_{e}$, and traversed distance $\Delta L$; (ii) calculate the neutron flux. The neutron flux to the direction of observation is incremented according to the differential cross section for neutron production; (iii) pseudo-collision and change direction. A new direction $\Omega(\theta$, $\varphi)$ is assigned by selecting new azimuthal and polar angles. The polar angle $\theta$ is sampled from the multiple-scattering distribution function ${ }^{33}$ and the azimuthal angle $\varphi$ is chosen randomly in the interval $[0-\pi]$; and (iv) move the deuteron to a new position. The above algorithm is applied until the ion energy becomes less than a prescribed cut-off energy (typically, $1 \mathrm{keV}$ ).

\section{RESULTS AND DISCUSSION}

Neutron spectra averaged over several shots $\left(I=2.6 \times 10^{19} \mathrm{~W} \mathrm{~cm}^{-2}\right.$ ) onto bulk CD targets for each of the neutron detector directions are shown in Figure 3(a). For comparison, the simulated neutron spectra are shown in Figure 3(b) as well as the simulated deuteron spectra in the inset. Scattering within shielding has been calculated to alter the apparent ToF spectrum by causing an energy down-shift of $0.05-0.15 \mathrm{MeV}$ as well as a spreading of $0.2-0.4 \mathrm{MeV}$ in the energy range recorded. In the forward directions, $\theta=10^{\circ}$ and $33^{\circ}$, the central energy was close to the expected 2.45 $\mathrm{MeV}$ and at high laser intensities, the peak neutron energy is greater than $2.45 \mathrm{MeV}$. However, in the backwards direction, not only was the yield much lower but also the energy had been downshifted. For beam-target interactions, the neutron energies are expected to be up-shifted or down-shifted in the lab frame depending on the incident deuteron energy and direction ${ }^{6}$ which explained the relative down-shift in neutron 

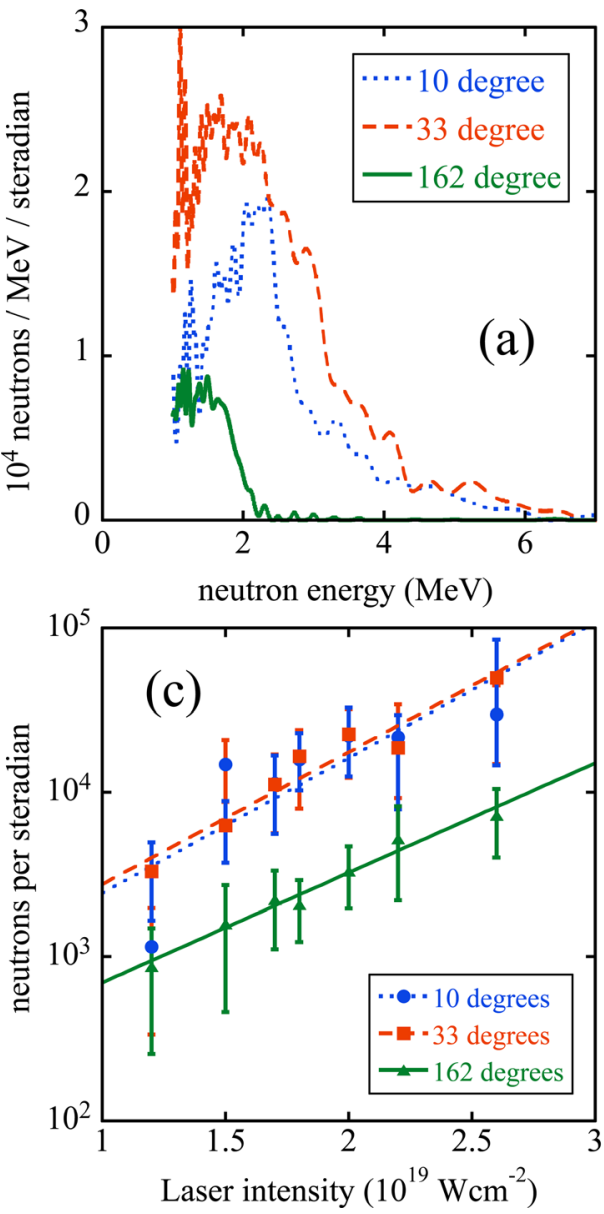
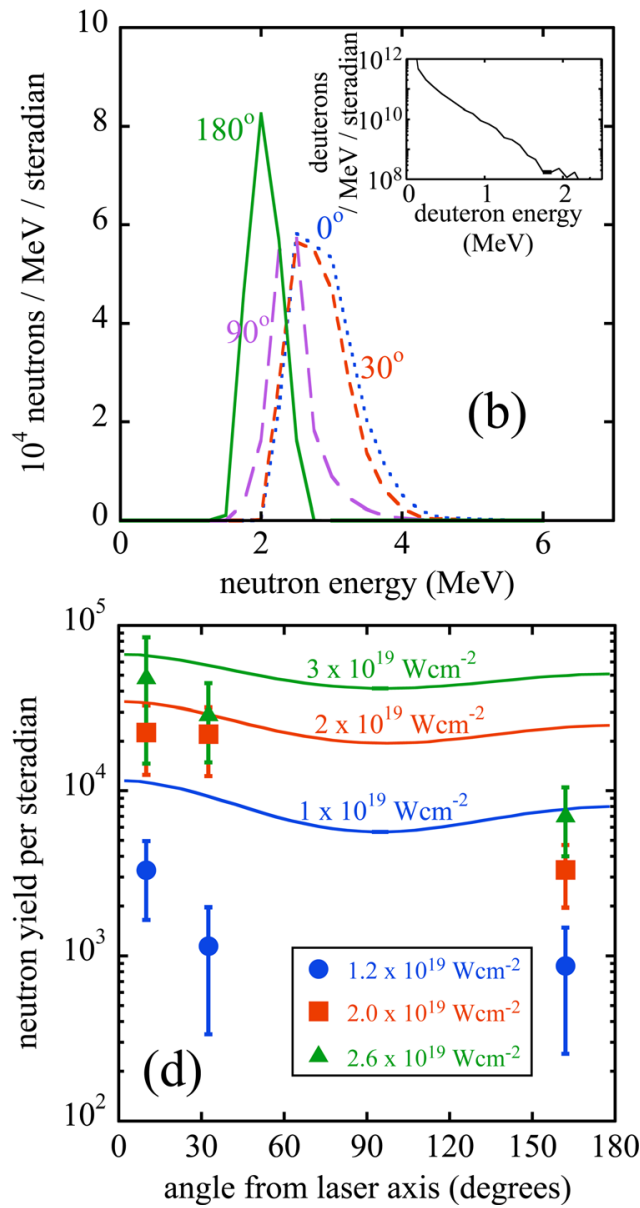

FIG. 3. (Color online) Bulk CD target data. Neutron spectra from (a) the experiment $\left(I=2.6 \times 10^{19} \mathrm{~W} \mathrm{~cm}^{-2}\right)$ and (b) the simulation $\left(I=3 \times 10^{19} \mathrm{~W}\right.$ $\mathrm{cm}^{-2}$ ), the inset in (b) is the simulation deuteron spectra. (c) The number of neutrons per steradian for different laser intensities. The dashed lines show exponential fits to the data. (d) Angular dependance of the neutrons per steradian. The stopping power is adjusted according to the averaged electron temperature from the PIC simulations in the neutron production calculation. The solid lines are from the FSA simulations. Note that the angles are given with respect to the laser axis, which is $22.5^{\circ}$ to the target normal in the experiment. energies observed in $\theta=162^{\circ}$ and also the peak up-shift at high intensity in the forward directions.

By integrating over the energy range of $1-4 \mathrm{MeV}$, the yield per steradian in each detector direction was deduced for each laser intensity, as shown in Figure 3(c). There is a steady exponential increase in neutron yield with laser intensity. This was consistent with the measurements of Disdier et $a l .{ }^{3}$ where the neutron emission is found to be strongly correlated to the transfer of laser energy into hot electrons. Note that this transfer of energy into hot electrons not only will influence the ion acceleration but also will affect the background plasma density in the target. The neutron yield versus angle for different laser intensities are shown in Figure 3(d) and compared with the FSA simulation data. The experiment shows a slightly stronger preference for neutron emission in the forward direction compared with the backward direction when compared with the simulations. This could be because the simulations only account for a temperature gradient into the target and not in the radial direction, which could influence the directionality of the neutrons further.

From the P-C targets, significantly fewer neutrons were measured, with most shots showing no discernible signal. Shown in Figures 4(a) and 4(c) are the neutron and deuteron spectra taken with a peak vacuum laser intensity of $1.9 \times 10^{19} \mathrm{~W} \mathrm{~cm}^{-2}$ onto a $13 \mu \mathrm{m}$ Mylar target with both sides having a CD coating. The measured deuteron spectra for this shot had an unusually high maximum energy (Figure 4(c)) and beam energy for this series of shots providing an explanation for the good neutron signal. The simulated neutron spectra for a laser intensity of $2 \times 10^{19} \mathrm{~W} \mathrm{~cm}-2$ are shown in Figure 4(b) and illustrate the expected preferential beaming of the high energy neutrons in the $0^{\circ}$ direction.

Figure 5 shows the increase in neutron yield with laser intensity. The measured neutron yield from RSA at $0^{\circ}$ shows very strong, almost exponential increase with laser intensity, consistent with the trend observed in previous measurements. ${ }^{3,9}$ The modeled neutron yield per steradian at $0^{\circ}$ is shown for both the RSA (solid squares) and the FSA (circles). The yields for both the cold target with bound electrons (hollow circles) and for the heated target (solid circles) are shown. If the target heating and consequent range enhancements are neglected, the yield increase was sublinear (dotted line), contrary to the experimental data. The inclusion of target heating in the stopping power leads to a qualitative improvement of the dependence of yield versus laser intensity. Nonetheless, below laser intensity $2.6 \times 10^{19} \mathrm{~W}$ $\mathrm{cm}^{-2}$, the simulated yield appears to be an overestimate, whereas at intensities above $2.6 \times 10^{19} \mathrm{~W} \mathrm{~cm}^{-2}$, the simulations underestimate the experimental neutron yield. This demonstrates that the calculation can be very sensitive to the assumed background temperature, but also that neutron yields could be significantly improved by increasing the background plasma temperature. Also, note that the temperature gradient is only in one-dimension (into the target) in the numerical model. But if the temperature gradient also had a radial dependance, deuterons with the same energy would be 

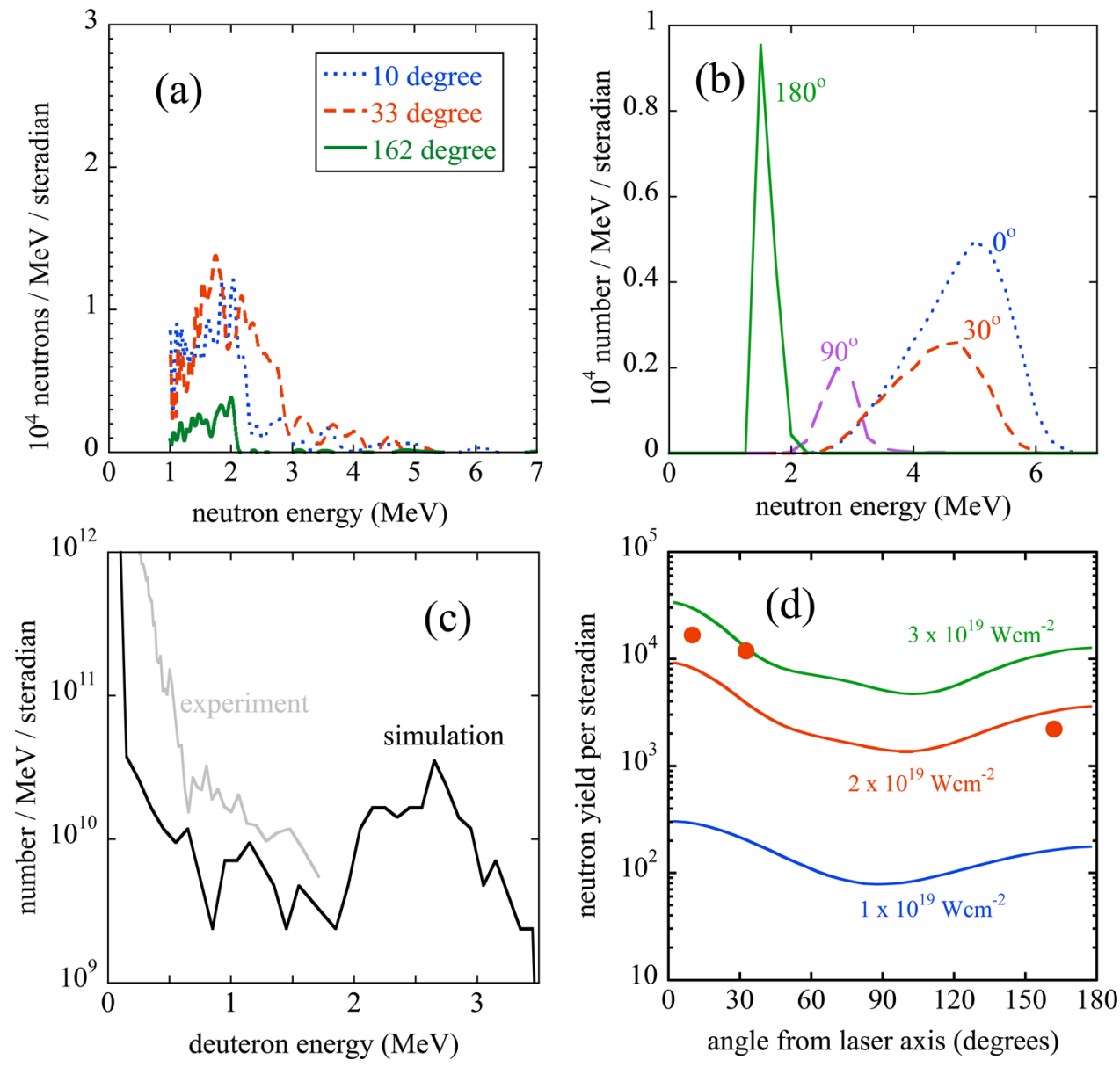

FIG. 4. (Color online) P-C target data. Neutron spectra from (a) the experiment $\left(I=1.9 \times 10^{19} \mathrm{~W} \mathrm{~cm}^{-2}, 13 \mu \mathrm{m}\right.$ Mylar foil coated on the both surfaces with $\mathrm{CD})$ and (b) the simulation $\left(I=2 \times 10^{19}\right.$ $\mathrm{W} \mathrm{cm}^{-2}$ ). (c) The experimental and simulated deuteron spectra. (d) The angular dependance on total neutron yield for the RSA simulations. The stopping power for a bound electron target is assumed for the neutron production calculation. The data points are from the experiment. Note that the angles are given with respect to the laser axis, which is $22.5^{\circ}$ to the target normal in the experiment. able to travel further into the target than in the radial direction and a possible enhancement in the collimation of the generated neutron beam could be produced. At low intensities, FSA performs best due to the large number of lower energy deuterons that can be accelerated. However, the RSA shows a stronger dependance with intensity and likely gener-

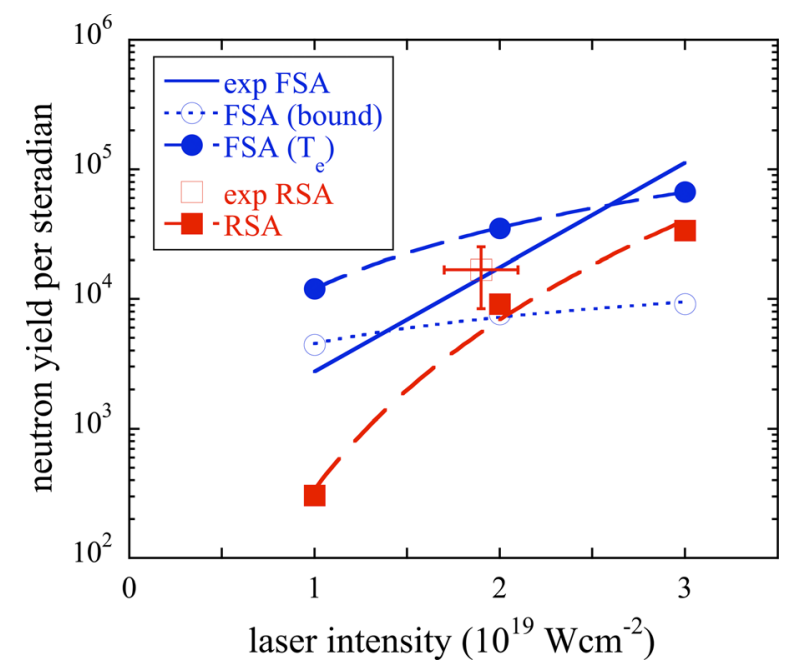

FIG. 5. (Color online) Simulated neutron yield at $0^{\circ}$ dependance on laser intensity for FSA (for both bound electron and heated target stopping powers, labeled bound and $T_{e}$, respectively) and RSA (bound electron stopping power). The line labeled exp FSA shows the experimental $0^{\circ}$ trend (Figure 3(c)) and the pitcher-catcher experimental result is also plotted (label exp RSA). ate higher fluxes than the FSA at higher intensities, particularly with regard to the generation of high energy neutrons which may be important for some applications. A study of how the contamination layer affects the deuteron acceleration shows how the suppression of the deuteron acceleration is more pronounced at lower intensities. ${ }^{28}$ Scaling the experiment to higher intensities, it would therefore be expected that the P-C target would produce a high energy more collimated neutron beam than the bulk CD target. Further improvement of the P-C scheme might be expected if the catcher target was pre-heated, although this may be challenging to realize for a large catcher area.

\section{SUMMARY}

In conclusion, experiments using a laser intensity in the range $(1-3) \times 10^{19} \mathrm{~W} \mathrm{~cm}^{-2}$ find that a bulk CD target was much more efficient than a pitcher-catcher target for neutron production. These are the first direct comparison of these neutron production mechanisms. Due to the high background temperature in the bulk target, the deuterons experience a reduced stopping power allowing them to travel further and therefore are more likely to undergo a $\mathrm{d}(d, n)-{ }^{3} \mathrm{He}$ fusion reaction, increasing the neutron yield. The P-C scheme was less effective because deuteron acceleration was less efficient from TNSA, the rear side deuteron acceleration was suppressed by any proton rich contamination layer, and because the catcher target was cold. However, the P-C method has more potential to yield higher fluxes of energetic 
neutrons and to create a more collimated neutron beam as it naturally selects a collimated ion beam. Improvements to the P-C scheme could be made through target cleaning to increase the deuteron acceleration efficiency, and by using a hot plasma catcher to increase propagation length and hence the reaction probability. The use of other neutron generating reactions, such as ${ }^{7} \mathrm{Li}(p, n)$ and ${ }^{7} \mathrm{Li}(d, n)$, which have larger cross-sections for neutron production, could also be used for either the bulk target or P-C configurations.

\section{ACKNOWLEDGMENTS}

This work was supported by the Defense Threat Reduction Agency (DTRA) and the Naval Research Laboratory (NRL). The authors thank Dr. M. R. Hartman for assistance with the d-d neutron generator.

${ }^{1}$ G. Pretzler, A. Saemann, A. Pukhov, D. Rudolph, T. Schätz, U. Schramm, P. Thirolf, D. Habs, K. Eidmann, G. D. Tsakiris, J. Meyer-ter-Vehn, and K. J. Witte, Phys. Rev. E 58, 1165 (1998).

${ }^{2}$ P. A. Norreys, A. P. Fews, F. N. Beg, A. R. Bell, A. E. Dangor, P. Lee, M. B. Nelson, H. Schmidt, M. Tatarakis, and M. D. Cable, Plasma Phys. Controlled Fusion 40, 175 (2004).

${ }^{3}$ L. Disdier, J.-P. Garçonnet, G. Malka, and J.-L. Miquel, Phys. Rev. Lett. 82, 1454 (1999).

${ }^{4}$ T. Ditmire, J. Zweiback, V. P. Yanovsky, T. E. Cowan, G. Hays, and K. B. Wharton, Nature (London) 398, 489 (1999).

${ }^{5}$ K. W. Madison, P. K. Patel, D. Price, A. Edens, M. Allen, T. E. Cowan, J. Zweiback, and T. Ditmire, Phys. Plasmas 11, 270 (2004).

${ }^{6}$ N. Izumi, Y. Sentoku, H. Habara, K. Takahashi, F. Ohtani, T. Sonomoto, R. Kodama, T. Norimatsu, H. Fujita, Y. Kitagawa, K. Mima, K. A. Tanaka, and T. Yamanaka, Phys. Rev. E 65, 036413 (2002).

${ }^{7}$ S. Fritzler, Z. Najmudin, V. Malka, K. Krushelnick, C. Marle, B. Walton, M. S. Wei, R. J. Clarke, and A. E. Dangor, Phys. Rev. Lett. 89, 165004 (2002).

${ }^{8}$ S. Karsch, S. Düsterer, H. Schwoerer, F. Ewald, D. Habs, M. Hegelich, G. Pretzler, A. Pukhov, K. Witte, and R. Sauerbrey, Phys. Rev. Lett. 91, 015001 (2003).

${ }^{9}$ H. Habara, R. Kodama, Y. Sentoku, N. Izumi, Y. Kitagawa, K. A. Tanaka, K. Mima, and T. Yamanaka, Phys. Rev. E 70, 046414 (2004).

${ }^{10}$ G. M. Petrov and J. Davis, Plasma Phys. Controlled Fusion 50, 015004 (2008).

${ }^{11}$ E. L. Clark, K. Krushelnick, J. R. Davies, M. Zepf, M. Tatarakis, F. N. Beg, A. Machacek, P. A. Norreys, M. I. K. Santala, I. Watts, and A. E. Dangor, Phys. Rev. Lett. 84, 670 (2000).

${ }^{12}$ A. Maksimchuk, S. Gu, K. Flippo, D. Umstadter, and V. Yu. Bychenkov, Phys. Rev. Lett. 84, 4108 (2000).

${ }^{13}$ L. O. Silva, M. Marti, J. R. Davies, R. A. Fonseca, C. Ren, F. S. Tsung, and W. B. Mori, Phys. Rev. Lett. 92, 015002 (2004).

${ }^{14}$ C. L. Ellison and J. Fuchs, Phys. Plasmas 17, 113105 (2010).

${ }^{15}$ S. P. Hatchett, C. G. Brown, T. E. Cowan, E. A. Henry, J. S. Johnson, M. H. Key, J. A. Koch, A. B. Langdon, B. F. Lasinski, R. W. Lee, A. J.
Mackinnon, D. M. Pennington, M. D. Perry, T. W. Phillips, M. Roth, T. C. Sangster, M. S. Singh, R. A. Snavely, M. A. Stoyer, S. C. Wilks, and K. Yasuike, Phys. Plasmas 7, 2076 (2000).

${ }^{16}$ L. Willingale, G. M. Petrov, A. Maksimchuk, J. Davis, R. R. Freeman, T. Matsuoka, C. D. Murphy, V. M. Ovchinnikov, L. Van Woerkom, and K. Krushelnick, Plasma Phys. Controlled Fusion 53, 014011 (2011).

${ }^{17}$ L. J. Perkins, B. G. Logan, M. D. Rosen, M. D. Perry, T. Diaz de la Rubia, N. M. Ghoniem, T. Ditmire, P. T. Springer, and S. C. Wilks, Nucl. Fusion 40, 1 (2000).

${ }^{18}$ K. L. Lancaster, S. Karsch, H. Habara, F. N. Beg, E. L. Clark, R. Freeman, M. H. Key, J. A. King, R. Kodama, K. Krushelnick, K. W. D. Ledingham, P. McKenna, C. D. Murphy, P. A. Norreys, R. Stephens, C. Stöeckl, Y. Toyama, M. S. Wei, and M. Zepf, Phys. Plasmas 11, 3404 (2004).

${ }^{19}$ J. M. Yang, P. McKenna, K. W. D. Ledingham, T. McCanny, L. Robson, S. Shimizu, R. P. Singhal, M. S. Wei, K. Krushelnick, R. J. Clarke, D. Neely, and P. A. Norreys, J. Appl. Phys. 96, 6912 (2004).

${ }^{20}$ D. P. Higginson, J. M. McNaney, D. C. Swift, T. Bartal, D. S. Hey, R. Kodama, S. Le Pape, A. Mackinnon, D. Mariscal, H. Nakamura, N. Nakanii, K. A. Tanaka, and F. N. Beg, Phys. Plasmas 17, 100701 (2010).

${ }^{21}$ J. Davis, G. M. Petrov, Tz. Petrova, L. Willingale, A. Maksimchuk, and K. Krushelnick, Plasma Phys. Controlled Fusion 52, 045015 (2010).

${ }^{22}$ V. S. Belyaev, V. I. Vinogradov, A. P. Matafonov, S. M. Rybakov, V. P. Krainov, V. S. Lisitsa, V. P. Andrianov, G. N. Ignatiev, V. S. Bushuev, A. I. Gromov, A. S. Rusetsky, and V. A. Dravin, Phys. At. Nucl. 72, 1077 (2009).

${ }^{23}$ T. Žagar, J. Galy, J. Magill, and M. Kellett, New J. Phys. 7, 253 (2005).

${ }^{24}$ A. Youssef and R. Kodama, Nucl. Fusion 50, 035010 (2010).

${ }^{25}$ J. Fuchs, Y. Sentoku, S. Karsch, J. Cobble, P. Audebert, A. Kemp, A. Nikroo, P. Antici, E. Brambrink, A. Blazevic, E. M. Campbell, J. C. Fernández, J.-C. Gauthier, M. Geissel, M. Hegelich, H. Pépin, H. Popescu, N. Renard-LeGalloudec, M. Roth, J. Schreiber, R. Stephens, and T. E. Cowan, Phys. Rev. Lett. 94, 045004 (2005).

${ }^{26}$ R. A. Forster, L. J. Cox, R. F. Barrett, T. E. Booth, J. F. Briesmeister, F. B. Brown, J. S. Bull, G. C. Geisler, J. T. Goorley, R. D. Mosteller, S. E. Post, R. E. Prael, E. C. Selcow, and A. Sood, Nucl. Instrum. Methods Phys. Res. B 213, 82 (2004).

${ }^{27}$ G. M. Petrov and J. Davis, Comput. Phys. Commun. 179, 868 (2008).

${ }^{28}$ G. M. Petrov, L. Willingale, J. Davis, Tz. Petrova, A. Maksimchuk, and K. Krushelnick, Phys. Plasmas 17, 103111 (2010).

${ }^{29}$ J. Davis and G. M. Petrov, Plasma Phys. Controlled Fusion 50, 065016 (2008).

${ }^{30}$ T. A. Mehlhorn, J. Appl. Phys. 52, 6522 (1981).

${ }^{31}$ J. F. Ziegler, J. Appl. Phys. 85, 1249 (1999).

${ }^{32}$ J. P. Biersack and L. G. Haggmark, Nucl. Instrum. Methods 174, 257 (1980).

${ }^{33}$ J. D. Jackson, Classical Electrodynamics (Wiley, New York, 1975), p. 647.

${ }^{34}$ J. M. Dawson, H. P. Furth, and F. H. Tenney, Phys. Rev. Lett. 26, 1156 (1971).

${ }^{35}$ F. Santini, Nucl. Fusion 46, 225 (2006).

${ }^{36}$ M. Sherlock, S. J. Rose, and A. P. L. Robinson, Phys. Rev. Lett. 99, 255003 (2007).

${ }^{37}$ J. J. Honrubia, A. A. Antonicci, and D. Moreno, Laser Part. Beams 22, 129 (2004) 